\title{
Piezo-Assisted Turbinoplasty: A Novel Rapid and Safe Technique
}

\author{
Enrico Robotti, MD ${ }^{1}$ Ali Khazaal, MD ${ }^{2}$ Francesco Leone, MD ${ }^{1}$ \\ 1 Private Practice-Plastic Surgery, Bergamo, Italy \\ ${ }^{2}$ Bagdad-ORL, Bagdad, Iraq \\ Address for correspondence Francesco Leone, MD, Private Practice- \\ Plastic Surgery, Via Pelandi 27, Bergamo 24125, Italy \\ (e-mail: dott.leone@hotmail.com).
}

Facial Plast Surg 2020;36:235-241.
Abstract Keywords
- turbinate hypertrophy
- turbinates
- turbinoplasty
- nasal obstruction
- piezoelectric

Consensus is still lacking on the ideal treatment of turbinate hypertrophy concurrent with rhinoseptoplasty. A novel technique of turbinoplasty consisting of incision-bone fracturing by the use of piezoelectric technique-intramucosal microcauterizationlateralization is described in detail. A series of 157 consecutive patients is reviewed with a maximum follow-up of one year. The technique is fast and easy and allows predictability in avoiding postoperative bleeding and preventing remedialization of the lateralized turbinates. Due to the technology required, its use is suggested especially when piezo is employed during other steps of rhinoseptoplasty.

Lack of consensus and mixed results are the most striking features when reviewing the existing literature concerning treatment of inferior turbinate hypertrophy (ITH).$^{1-6}$ This is mostly due to the shortage of perspective, large number, studies with inadequate long-term follow-up, combined with the fact that multiple variables, which cannot be easily evaluated separately, are at play both intraoperatively and in functional assessment postoperatively. A wide variety of medical and surgical alternatives, alone or combined, thus exist in the treatment of ITH, without their efficacy being either measured or proven. Surgical techniques differ markedly regarding tissue excision or preservation. This leads to uncertainty not only on the treatment of turbinate hypertrophy per se, but also on how to treat hypertrophic turbinates in the common setting of a rhinoseptoplasty where hypertrophy of the contralateral turbinate to the concave side of the deviated septum is treated together with septoplasty. Some facts, however, are generally agreed upon especially in the recent literature:

1. There is general concert on the rationale to combine septoplasty with turbinoplasty when treating septal deviation. This is done both to reduce hypertrophy of the contralateral turbinate, which expands and "curls" around the deviation, as well as to allow enough space for the septum to be repositioned in the midline $2,7-9$

published online December 31, 2019
Issue Theme Facial Plastic Surgery Original Research; Guest Editors: Anthony P. Sclafani, MD, MBA, FACS, and Alwyn D'Souza, MBBS, FRCS Eng, FRCS (ORL-HNS)
2. It is well known that both mucosal and bone hypertrophy usually coexist in turbinate hypertrophy. This leads to the frequent combination of procedures that treat both mucosa and bone in combination. ${ }^{2,10}$

3. There seems to be consensus on the fact that bone matters more, especially in compensatory hypertrophy of the inferior turbinate. ${ }^{11,12}$

4. Most studies concur on the fact that lateralization, which implies bone out-fracturing rather than bone removal, is effective and that its results can be durable, ${ }^{13-17}$ although some disagree on the long-term outcome. ${ }^{6}$ Such efficacy seems increased when combined with other treatments addressed to mucosal hypertrophy. ${ }^{3,7,13,17-19}$ Significantly, submucosal resection combined with lateralization results seem to provide excellent results long-term. ${ }^{20,21}$

5. A common complication of turbinoplasty is intraoperative and postoperative bleeding. ${ }^{1,6}$ Thus, techniques that reduce bleeding should be preferred. Submucosal turbinate resection may cause bleeding.

Considering all the above, it would stand to reason that the ideal method for ITH would be one that aims to prevent bleeding, is fast to perform and implies bone fracturing and lateralization, as well as coexisting treatment of the hypertrophic mucosa.
Copyright (c 2020 by Thieme Medical Publishers, Inc., 333 Seventh Avenue, New York, NY 10001, USA. Tel: +1(212) 760-0888.
DOI https://doi.org/ 10.1055/s-0039-3401803. ISSN $0736-6825$. 


\section{Relevant Anatomy}

Most studies highlight the importance of bony as well as mucosal contribution to ITH. ${ }^{2,10}$ A prevalence of bone versus mucosa enlargement has been demonstrated by several authors. ${ }^{10-12}$ Patients with septal deviation have compensatory ITH which is mainly due to the expansion of the bone rather than the mucosa. ${ }^{10,12}$

The usual shape of the turbinate bone is uncinate, with the bone projecting from the lateral nasal bony wall at an acute angle. Understanding the blood supply of the inferior turbinate is important regarding its relationship to potential bleeding: the sphenopalatine artery, after exiting from its foramen on the lateral nasal wall, becomes the posterior lateral nasal artery which ends up into the two main inferior turbinate and middle turbinate branches. Importantly, the inferior turbinate branch generally runs in close relation to the bone of the inferior turbinate medially, until it ends on the anterior part of the turbinate while still maintaining its relatively large diameter Although anatomical variations exist and a further contribution to the inferior turbinate branch of the sphenopalatine artery may originate from the descending palatine artery, it is important to note that in the vast majority of specimens of most studies the relatively large branches which supply the inferior turbinate enter the turbinate posteriorly and then run medially, adherent to the bone or travelling in a canal within the bone. ${ }^{22-24}$

The close relationship between the inferior turbinate branch and the turbinate bone well explains why bone resection/trimming during turbinate reduction may lead to even considerable bleeding. Most peri- and postoperative problems that occur while treating ITH are indeed related to hemorrhage, hence the obvious merits of a method which will prevent such occurrence.

\section{Cone-beam Analysis}

A computed tomography (CT) scan is a well-recognized, objective, non-operator-dependent, method of assessing turbinate hypertrophy before surgery. Although several studies have also used CT for postoperative evaluation of results, ${ }^{8,12-15,25,26}$ for instance, following outfracture procedures, it remains difficult to justify a routine execution of the procedure postoperatively in clinical practice.

As well as defining dimensions, a CT scan can also discriminate and accurately measure the bone versus mucosa component of the inferior turbinate. A definite further advantage is the use of the cone-beam CT scan (CBCT), as favored extensively by the senior author over the last years in his rhinoplasty practice. CBCT is still unfamiliar to most surgeons performing rhinoplasty, both plastic surgeons and otolaryngologists, while it is better known in the dental and maxillofacial community, where it finds, for instance, uniform applications for treatment, planning, and diagnosis in implant dentistry. ${ }^{27,28}$ With a quantity of delivered radiation about the seventh part of a conventional CT scan CBCT is a user-friendly, quick technique which can be extrapolated with many advantages to rhinoplasty planning.

What CBCT can easily do is to essentially provide a useful roadmap to surgery regarding the following: studying septum deformities and deviations; studying the nasal bones regarding their shape, symmetry, and thickness; assessing the thickness of the skin; and demonstrating the length and shape of the bony cap together with the underlying extension of the upper laterals. Although outside the scope of this article (and object of a forthcoming publication by the senior author), it has to be noted that CBCT in rhinoseptoplasty has the great advantage of allowing viewing the axial coronal and sagittal planes at once, running and scrolling all three in parallel, as well as providing a striking quality of three-dimensional (3D) imaging. Different setups in 3D reconstruction permit to highlight progressively bone versus soft tissue, thus demonstrating contour details and irregularities as connected to the underlying bone. CBCT, like any CT scan, is also easily employed to study both the middle turbinate, regarding the presence and shape of concha bullosa, as well as the inferior turbinate regarding its measurable volume and percentage of bone versus mucosa. This is obviously an element that may guide the decision for turbinoplasty with or without fracturing the uncinate bone.

CBCT, as compared with the conventional CT, causes the patient no inconvenience (is done standing in a few minutes) and has very few if any drawbacks, these being minimal radiation and a very limited cost.

\section{Surgical Technique (see - Video 1)}

\section{Video 1}

The video shows a three-dimensional representation built on the cone-beam CT images and the step-bystep turbinoplasty surgical technique. Online content is viewable at: https://www.thieme-connect.com/ products/ejournals/html/10.1055/s-0039-3401803.

Analysis. The decision for turbinoplasty is based primarily on clinical examination and CBCT scan. Although factors contributing to functional deficiency in each patient are multiple and it is not easy to define the specific contribution of each, the usual setting is one of a deviated septum with contralateral turbinate enlargement. Preoperatively, all patients undergo a CBCT scan. As well as being a roadmap to many elements relevant to rhinoseptoplasty as noted earlier, the CBCT will allow dimensional assessment of an enlarged turbinate, demonstrate compensatory hypertrophy in deviated septum, and show the percentage of bone versus mucosa.

Anesthesia. All patients are operated under general hypotensive anesthesia with endotracheal intubation. Turbinoplasty is never performed per se in the senior author's practice, but only as an adjunct to a rhinoseptoplasty, consistently done by the open approach. Generally, after completing the septoplasty by in situ or extracorporeal technique, if necessary, infiltration of the inferior turbinate is done from anterior to posterior with $1 \%$ lidocaine with 1 to 100,000 epinephrine administered by a dental Carpule syringe. 


\section{Incision and Limited Tunneling at Bony Edge}

Turbinoplasty is usually performed by the senior author following completion of dorsal maneuvers, including hump reduction, osteoplasty, and osteotomies. Septoplasty can be done before or after, depending on the circumstances: doing it before safeguards the septum from any accidental dislodgement during turbinoplasty, while doing it after is simpler because of the additional space gained on either side of the septum. Essentially, it depends on the complexity of the septoplasty.

A full-thickness incision measuring 5 to $8 \mathrm{~mm}$ through the anterior-inferior surface of the turbinate is made by a fine angled Microsurgical needle (E1651-Covidien Valleylab) in the cutting mode. The incision has just to be long enough to allow passage for the small elevator first, and then the insert to follow. Once the Bovie tip touches bone, it is withdrawn and a short tunnel is made between the mucosa and the inferior turbinate bone, by the use of a small sharp elevator (Micro Elevator 190-268, Marina Medical, Florida). This tunnel only extends a few millimeters distal to the bony edge.

\section{Piezo Osteotomy}

Next, the elevator is withdrawn and a long, angled or straight, piezo insert (UniVR 03600008 [-Fig. 1a] or MT91303600016 [-Fig. 1b]; Mectron) is carefully inserted into the tunnel. It doesn't much matter whether the insert is angled or straight, while the appropriate length matters. Essentially, the same insert used in other portions of the rhinoplasty is re-employed. This is either the same insert used by the senior author in most rhinoplasties for low to low osteotomies (angled: UniVR), or the insert used for posterior septoplasty and resection of vomerine deviations (straight: MT9-13).

The insert is then activated and moved in a direction from caudal to cephalic, essentially close to and perpendicular to the line of attachment of the turbinate bone to the lateral nasal wall, which is in fact the site of maximum angulation as shown in -Fig. 2a,b. This is different and distinct from the line of conventional (manual) outfracture, which occurs more

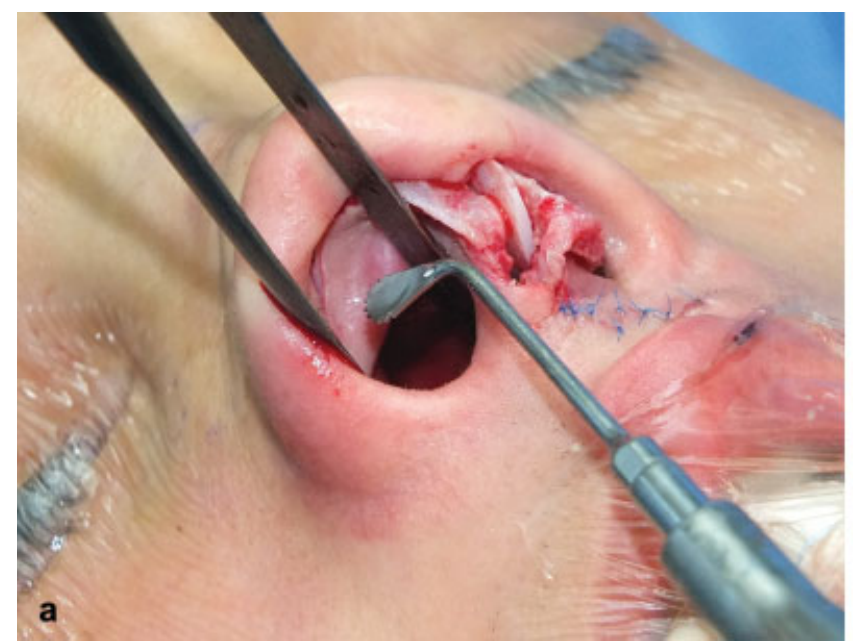

laterally, along the weakest point of the bone (-Fig. 3). One will usually feel initial bone resistance and then a clear give as the bone is entirely cut by the piezo insert where it angles. It is striking to note that at this point the inferior turbinate easily lateralizes passively, actually almost spontaneously. Occasionally, a proper break at the desired point doesn't happen, and one has to persist in finding further paths of bone resection by pushing the insert on a different trajectory, until the same result of "breaking a hinge," sometimes in more than one spot, is obtained. The bone is thus piecemealed, however, with an equally effective outcome.

\section{Submucosal Needle Microcauterization}

Next, the same Bovie tip cautery is introduced, submucosally along its full length, prevalently in the inferomedial portion of the turbinate. Coagulation current is used while the needle is withdrawn progressively, thus achieving a submucosal intraturbinal electric microcauterization which works with a "thermage" effect to reduce the soft tissue volume by fibrosis without the direct damage caused by surface electrocautery. Three or four passes are usually made.

\section{Finishing Maneuvers}

Once the steps mentioned before are completed, large Mayo scissors are introduced, placed flat on the turbinate, and used to complete lateralization of the turbinate. Some bleeding at the site of the initial full thickness incision is frequent and the edges are cauterized by bipolar cautery. No suturing is necessary. A Merocell sponge (Medtronic Inc.) is then inserted between turbinate and septum. When expanded, it will oppose any tendency to further bleeding by compression.

After the remaining tip portion of the rhinoplasty is completed and the incisions are closed, Doyle splints are routinely employed. A Vaseline gauze, impregnated with Bactroban ointment (GlaxoSmithKline plc.) is also routinely inserted between the Doyle splint and the turbinate on either side. In addition to splinting the septum, Doyle splints may also serve to help maintain the achieved lateralization of the inferior turbinates. The duration of the whole turbinoplasty is usually of two or three minutes for each turbinate. This is

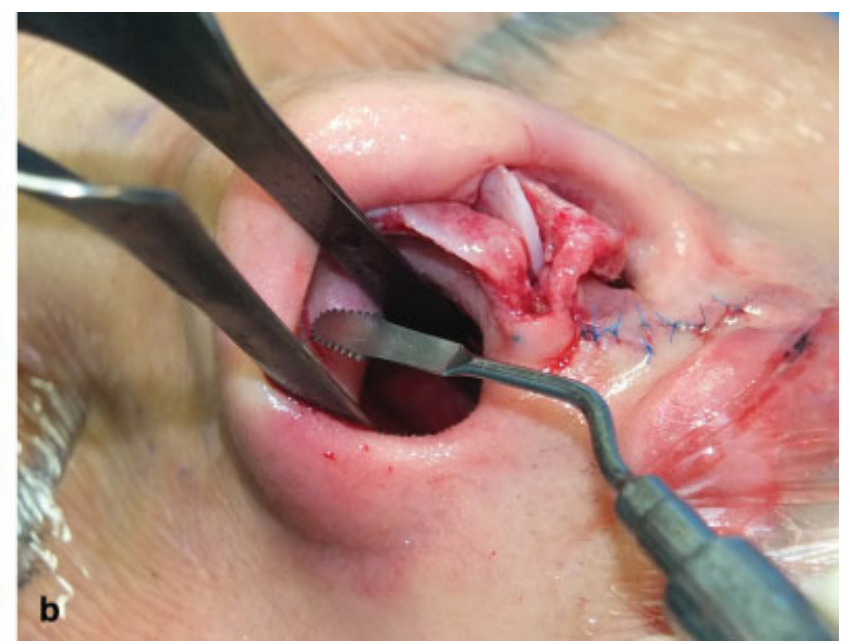

Fig. 1 The two piezo inserts usually employed (a) angled UniVR and (b) straight MT9-13. 

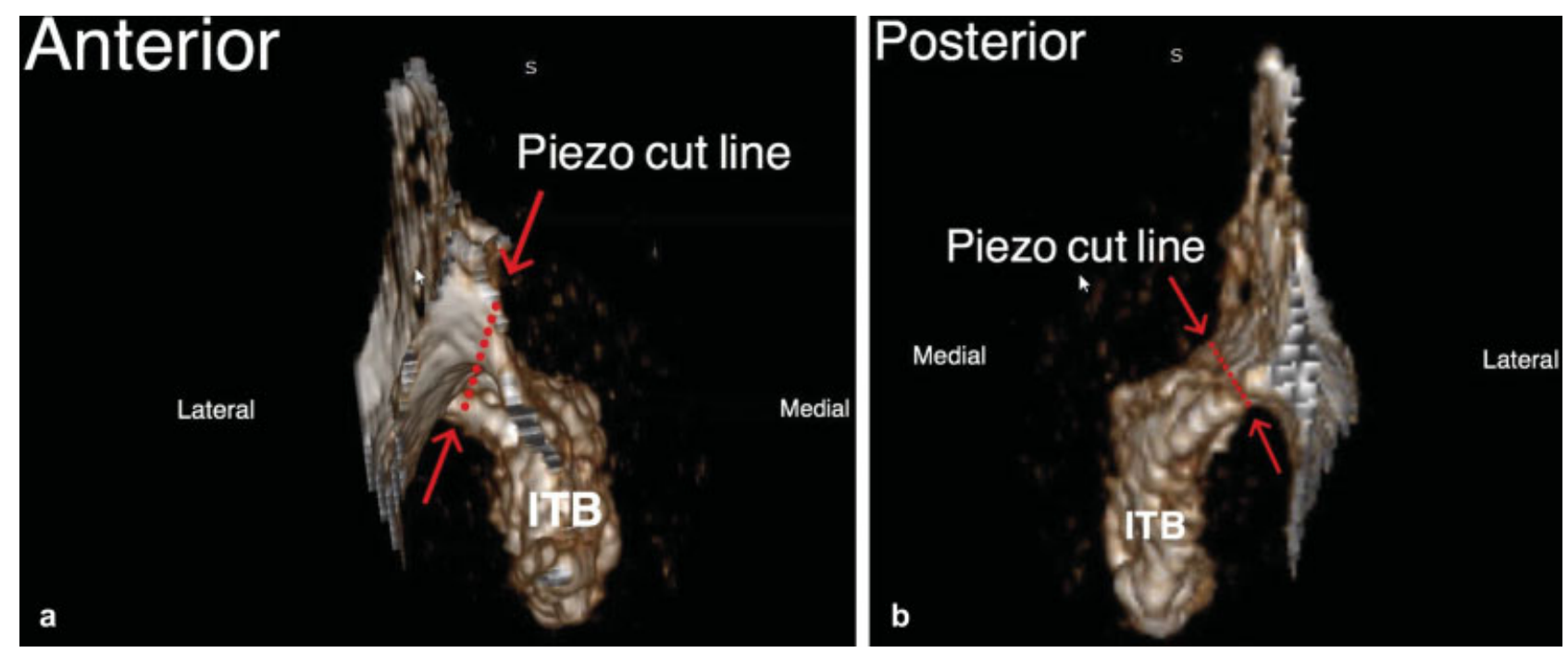

Fig. 2 Three-dimensional rendering of the right inferior turbinate bone illustrating the piezo cut line. (a) Anterior view. (b) Posterior view.

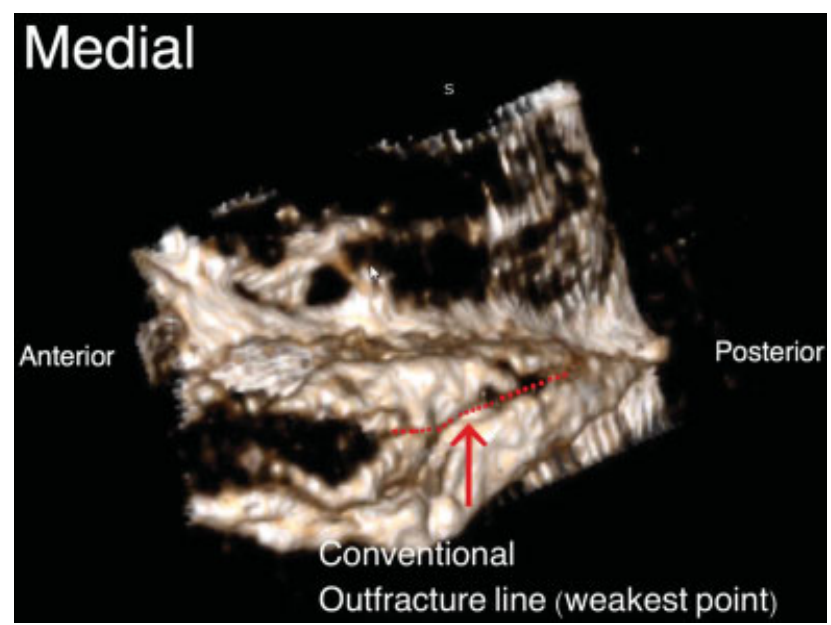

Fig. 3 Three-dimensional rendering of the inferior turbinate bone showing the line of the conventional outfracture along the weakest part of the bone (red dots).

negligible, especially when compared with the three hours of standard duration of a primary rhinoseptoplasty.

\section{Clinical Series and Results}

In this article, we present a case series of 157 consecutive patients, all operated in the same venue in a private setting by the senior author for rhinoseptoplasty concomitant with turbinoplasty using the technique described earlier, over a full year between February 2018 and February 2019. The study was conducted according to the declaration of Helsinki for biomedical research of human subjects. In no instance was turbinoplasty performed as a separate maneuver. Eighty-nine patients were females and 68 males, with age range from 16 to 73 years; 105 patients $(67 \%)$ were primaries and 52 (33\%) secondaries, with this designation generally indicating revision cases operated once or more previously, most frequently requiring rib grafting. This case series obviously excluded a minority of patients operated for rhinoseptoplasty over the same time span in which no turbinoplasty was performed (some secondaries where aggressive turbinectomy had been previously performed or the rare primaries with very small turbinates).

All patients underwent CT scan preoperatively, usually $\mathrm{CBCT}$, save for those that had had a conventional CT already done. Criteria for turbinoplasty were multiple:

1. Preoperative functional issue (reported breathing difficulty) as confirmed on clinical examination and CT-CBCT.

2. Concurrent correction of deviated septum with controlateral turbinate enlargement.

3. Prophylaxis, where a reduction of airway volume was anticipated.

It has to be noted that a low threshold was employed in deciding on turbinoplasty. Even in those patients who reported breathing satisfactorily preoperatively, turbinoplasty was usually performed "prophylactically" when narrowing and lowering of the bony vault was planned, unless the turbinates were especially small, in presence or absence of septal deviation.

All patients were seen between 3 to 6 and 9 to 12 months, within the postoperative visit related to rhinoseptoplasty. For those in the latter part of the study, follow-up was obviously limited between 9 to 6 and 3 months. Most patients complied with all their planned follow-up. Eleven patients did not return following the first follow-up at 3 months.

Postoperative outcomes pertinent to the study addressed breathing function, clinical examination, and assessment of complications.

Breathing function improvement was measured by a brief questionnaire, which was essentially a patient-reported outcome measure comparing preoperative with postoperative status. The simple question on postoperative check was "is your breathing improved, same, or worse?" Judging the efficacy by patient-reported outcome and perception of effectiveness may seem subjective, but it is a known fact that quantitative measurements of airflow do not necessarily 
correlate with what the patient perceives as effective improvement. ${ }^{1}$ In all patients breathing function had improved in those that had reported limitation beforehand, while it was perceived as unchanged or even improved in those who had good breathing before. This latter fact is interesting and may be due to the realization by the patient that the perceived "good breathing" preoperatively was actually not so when compared with the postoperative breathing. The difficulty is discriminating what variable made the greater difference, considering that many factors matter in relieving obstruction and that the effect of maneuvers on the septum, valves, and turbinates cannot be analyzed in isolation. In fact, some form of septoplasty was performed in over $80 \%$ of patients and valves and sidewall were often addressed, especially in secondaries, etc. No discrimination was thus attempted in understanding which factors contributed, and in which percentage, to improved breathing. Repeat CT scan, which would have been an ideal tool, was considered not justifiable in this patient population.

Clinical examination was undertaken by a long and thin speculum. Although no direct measurement of the distance between septum and medial turbinate edge was done, it was commonly observed that the position of the turbinate remained generally lateralized. Save for some instances in which mucosal hypertrophy was still evident (or possibly had already recurred), it was our constant impression that lateralization was maintained. Obviously, these findings had to be tempered by and related with the changes due to septoplasty.

Noted complications: No postoperative bleeding occurred in any of the patients. Postoperative bleeding was defined as any occurrence of bleeding requiring operative intervention with cauterization or ligation. Thus, some occasions of minor oozing occurring on the following morning postoperatively when the Vaseline gauze between Doyle and turbinate was removed were not considered as "postoperative bleeding." They were treated by simply replacing the Vaseline gauze for another day. In no instance purulence, foul drainage, prolonged crusting, rhinorrhea, and synechiae were noted at follow-up. No residual crusting was found at the three months postoperatively, although some patients had more crusting than others over the first two or three weeks.

\section{Discussion}

A wide variety of treatment options exist for the management of ITH, indicating a remarkable lack of consensus prevalently due to the corresponding lack of perspective and comparative studies with adequate follow-up, as well as to the multiple variables at play in the assessment of airway function. ${ }^{1-6}$ Obviously, turbinoplasty is very often performed during rhinoplasty, according to the commonly accepted logic of performing septoplasty together with reduction of the enlarged contralateral ITH. ${ }^{2,7-9,26}$ Interestingly, at least among plastic surgeons, conventional methods of turbinate reduction are generally preferred on the use of newer techniques. ${ }^{4}$
The goal of surgical treatment should be reducing the volume of the inferior turbinate while preserving function, and it stands to reason that an effective treatment should address both bony and mucosal components of ITH. ${ }^{1-3}$ Various studies implicate that bone matters more, especially in compensatory hypertrophy, together with the fact that some mucosal shrinkage should also spontaneously occur in compensatory ITH once the related septal deviation is corrected. ${ }^{2,10-12}$

General agreement exists on the fact that the surface mucosa itself should be spared as much as possible, and this emphasized the role of submucosal turbinoplasty, which is, however, a term that applies both to submucosal soft tissue reduction targeting the erectile tissue under the epithelium as well as submucosal bone removal. Submucosal turbinoplasty as described by Rohrich in 2001 and employed by the senior author for many years, consists in the sequence of incision, elevation-exposure of the conchal bone, extraction of bony fragments, out fracture, and cautery of the raw mucosal margins. ${ }^{7}$ Piecemeal bony excision, as commonly done by the use of a Takahashi forceps, is, however, prone to bleeding, mucosal tears, and subsequent prolonged crusting. This is due to the vascular anatomy of the inferior turbinate where the vessels travel in intimate relationship with the bone, as described earlier, and in fact occurred in the senior author's practice in multiple occasions in the past.

It thus seems logical that lateralization of the inferior turbinate has been recently favored by many authors as a simple technique that preserves function completely without any risk of bleeding. ${ }^{17}$ Lateralization is generally done by outfracture. Although the outcome of various studies was that the technique is effective with a durable reduction of angle and distance between the turbinate bone and the lateral wall, some authors noted the tendency of lateralized turbinate to recur in the long term. This essentially led to the procedure being used mainly as a complementary technique together with others, rather than a standalone method.,6,13,17

Interestingly, following their previous work on submucosal turbinoplasty, ${ }^{7}$ Rohrich et al later described closed microfracture of the turbinate bone inside an intact mucosal sac as the preferred method for treating ITH. ${ }^{16}$ Microfracturing and the related comminution was the key element they identified in reducing the risk of recurrence of the lateralized turbinates in the original position.

With these premises, it would make sense, while adopting the principle of avoiding extraction of bone and the related risk of bleeding, to adopt a technique which would reliably and permanently modify the bone architecture by precise resection while completely sparing soft tissue and mucosa. This would seem preferable to employing an elevator or a nasal speculum and causing possibly uncontrolled greenstick fractures by pressure only. Piezo can be helpful to this regards: through an incision measuring only a few millimeters, the insert can create a specific cut in the bony exactly at the line of maximum angulation, which is verified and planned preoperatively, depending on the CT or, preferably, CBCT imaging, with further help as well as of a possible 3D bone reconstruction. This complete linear cut is done with a long insert where the 

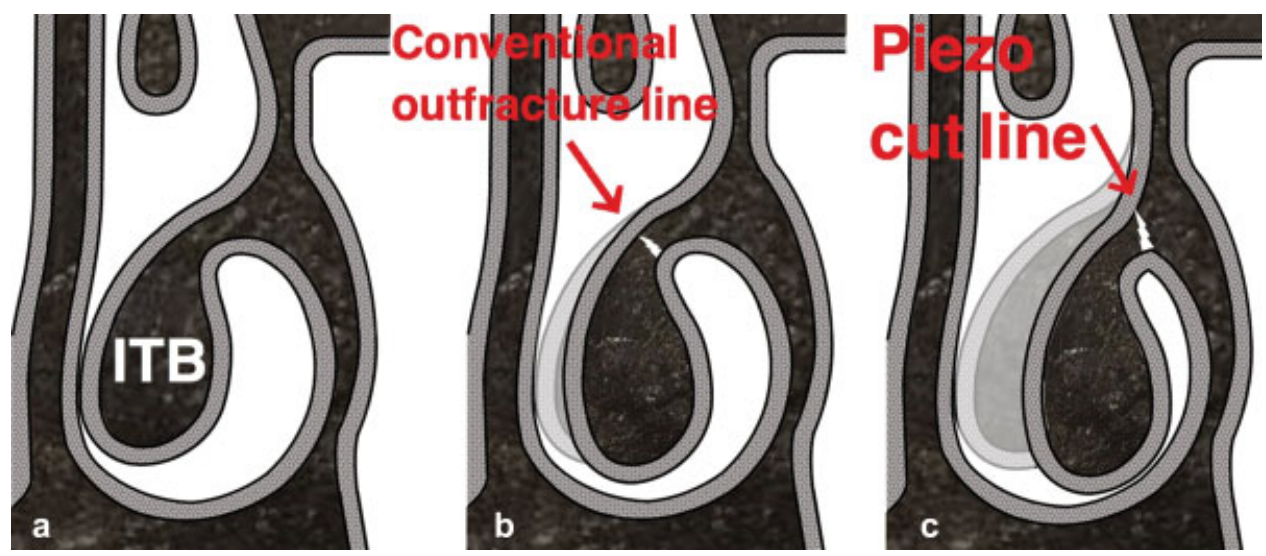

Fig. 4 Comparing techniques (conventional outfracture versus piezo lateralization) for the treatment of inferior turbinate hypertrophy in rhinoplasty patients.

uncinate bone angles off the nasal sidewall ( - Fig. $4 a-c$ ). If one is not sure that the direction of the insert and position of cut is at this spot, the angle of approach can be changed, and a new path of cut adopted. Not being a greenstick fracture but a true cut, its effect is stable and irreversible and the turbinate position has no risk of recurring. Bone is not removed, but its configuration is changed permanently.

Keeping Doyle splints for one week, as customary in rhinoseptoplasty, may help in further preventing any remedialization. ${ }^{2,16}$ Piezo technology, by definition, will spare soft tissue and vessels and has gained considerable momentum and widespread acceptance in rhinoplasty over the last few years especially for osteotomy and osteoplasty maneuvers. ${ }^{29}$ In the senior author's experience, piezo is used in multiple steps of rhinoplasty in every case, primary or secondary. It thus becomes logical to use the same insert that is employed for a lateral osteotomy or for posterior septal harvesting to also fracture the uncinate bone precisely, without causing any bleeding. It is to be noted that this is a different technique from the "sonic rhinoplasty" based on the use of an ultrasonic bone aspirator which emulsifiers and extracts bone. ${ }^{30}$

Additionally, on the premise stated earlier that bony and mucosal hypertrophy is concurrent in ITH, it also sounds reasonable to employ an adjunct treatment modality that, after bony hypertrophy is corrected, will then somewhat reduce the mucosa component, where it is thicker, that is, medially. This is why we add intramucosal microneedling with a unipolar electrode Bovie tip. The rationale is that intraturbinal thermocoagulation should cause less damage to mucosa than surface cautery, essentially inducing submucosal fibrosis. The "shrinkage" effect on the mucosa may of course well be temporary, but it will cause virtually no harm. - Table 1 summarizes the main considerations on the treatment of turbinates using piezoelectric technology.

The results described in this article need to be implemented by a longer term follow-up, and possibly by an objective method of assessing the stability of lateralization. Obviously, a repeat CBCT scan would be ideal, but this is hard to justify in the setting of a clinical practice.

\section{Conclusion}

The ideal surgical management of ITH should be efficient in relieving obstruction symptoms and kind to turbinate function. Piezo outfracturing of the turbinate bone combined with unipolar microcauterization is a safe, fast, easy, and effective technique for treating mild, moderate, and severe ITH without the risk of bleeding and prolonged crusting.

Table 1 Advantages and disadvantages

\begin{tabular}{|l|l|}
\hline Advantages & Disadvantages \\
\hline $\begin{array}{l}\text { Lateralization of the turbinates is not based on a greenstick fracture } \\
\text { but on a piezo-assisted cut through the uncinate bone at its angle. } \\
\text { This prevents recurrence. }\end{array}$ & The cost of the piezo equipment. \\
\hline The piezo insert is the same used in other parts of the rhinoseptoplasty. & $\begin{array}{l}\text { The technique may not be justifiable for } \\
\text { standalone turbinoplasty. }\end{array}$ \\
\hline The incision for access of the insert is limited. & \\
\hline $\begin{array}{l}\text { Piezo-assisted osteotomy of the uncinate bone } \\
\text { spares vessels close to the bone and prevents bleeding. }\end{array}$ & \\
\hline $\begin{array}{l}\text { Additional intramucosal unipolar cautery on fine needle may } \\
\text { help reduce mucosal component of inferior turbinate hypertrophy. }\end{array}$ & \\
\hline The technique is simple and fast. & \\
\hline No complications were noticed in the series studied. & \\
\hline
\end{tabular}


Surgeons already employing piezoelectric technique for different steps of rhinoseptoplasty should have a low threshold for performing this technique to correct ITH.

\section{Funding}

The authors received no financial support for publication of this article.

\section{Conflicts of Interest}

The authors declare no conflict of interest. No author receives any royalties from the manufacturers of the piezo machine and inserts depicted in this article.

\section{References}

1 Bergmark RW, Gray ST. Surgical management of turbinate hypertrophy. Otolaryngol Clin North Am 2018;51(05):919-928

2 Downs BW. The inferior turbinate in rhinoplasty. Facial Plast Surg Clin North Am 2017;25(02):171-177

3 Sinno S, Mehta K, Lee ZH, Kidwai S, Saadeh PB, Lee MR. Inferior turbinate hypertrophy in rhinoplasty: systematic review of surgical techniques. Plast Reconstr Surg 2016;138(03):419e-429e

4 Tanna N, Im DD, Azhar H, et al. Inferior turbinoplasty during cosmetic rhinoplasty: techniques and trends. Ann Plast Surg 2014;72(01):5-8

5 Hol MK, Huizing EH. Treatment of inferior turbinate pathology: a review and critical evaluation of the different techniques. Rhinology 2000;38(04):157-166

6 Jackson LE, Koch RJ. Controversies in the management of inferior turbinate hypertrophy: a comprehensive review. Plast Reconstr Surg 1999;103(01):300-312

7 Rohrich RJ, Krueger JK, Adams WP Jr, Marple BF. Rationale for submucous resection of hypertrophied inferior turbinates in rhinoplasty: an evolution. Plast Reconstr Surg 2001;108(02): 536-544, discussion 545-546

8 Jun BC, Kim SW, Kim SW, Cho JH, Park YJ, Yoon HR. Is turbinate surgery necessary when performing a septoplasty? Eur Arch Otorhinolaryngol 2009;266(07):975-980

9 Han JK, Stringer SP, Rosenfeld RM, et al. Clinical consensus statement: septoplasty with or without inferior turbinate reduction. Otolaryngol Head Neck Surg 2015;153(05):708-720

10 Berger G, Hammel I, Berger R, Avraham S, Ophir D. Histopathology of the inferior turbinate with compensatory hypertrophy in patients with deviated nasal septum. Laryngoscope 2000;110 (12):2100-2105

11 Rudes M, Schwan F, Klass F, Gassner HG. Turbinate reduction with complete preservation of mucosa and submucosa during rhinoplasty. HNO 2018;66(02):111-117

12 Egeli E, Demirci L, Yazýcý B, Harputluoglu U. Evaluation of the inferior turbinate in patients with deviated nasal septum by using computed tomography. Laryngoscope 2004;114(01):113-117

13 Lee DC, Jin SG, Kim BY, et al. Does the effect of inferior turbinate outfracture persist? Plast Reconstr Surg 2017;139(02):386e-391e

14 Aksoy F, Yıldırım YS, Veyseller B, Ozturan O, Demirhan H. Midterm outcomes of outfracture of the inferior turbinate. Otolaryngol Head Neck Surg 2010;143(04):579-584
15 Buyuklu F, Cakmak O, Hizal E, Donmez FY. Outfracture of the inferior turbinate: a computed tomography study. Plast Reconstr Surg 2009;123(06):1704-1079

16 Rohrich RJ, McKee D, Malafa M. Closed microfracture technique for surgical correction of inferior turbinate hypertrophy in rhinoplasty: safety and technical considerations. Plast Reconstr Surg 2015;136(05):607e-611e

17 Moss WJ, Lemieux AJ, Alexander TH. Is inferior turbinate lateralization effective? Plast Reconstr Surg 2015;136(05):710e-711e

18 Wolfswinkel EM, Koshy JC, Kaufman Y, Sharabi SE, Hollier LH Jr, Edmonds JL. A modified technique for inferior turbinate reduction: the integration of coblation technology. Plast Reconstr Surg 2010;126(02):489-491

19 Nassif Filho AC, Ballin CR, Maeda CA, Nogueira GF, Moschetta M, de Campos DS. Comparative study of the effects of submucosal cauterization of the inferior turbinate with or without outfracture. Rev Bras Otorrinolaringol (Engl Ed) 2006;72(01):89-95

20 Passàli D, Lauriello M, Anselmi M, Bellussi L. Treatment of hypertrophy of the inferior turbinate: long-term results in 382 patients randomly assigned to therapy. Ann Otol Rhinol Laryngol 1999;108(06):569-575

21 Larrabee YC, Kacker A. Which inferior turbinate reduction technique best decreases nasal obstruction? Laryngoscope 2014;124 (04):814-815

22 Padgham N, Vaughan-Jones R. Cadaver studies of the anatomy of arterial supply to the inferior turbinates. J R Soc Med 1991;84 (12):728-730

23 Orhan M, Midilli R, Gode S, Saylam CY, Karci B. Blood supply of the inferior turbinate and its clinical applications. Clin Anat 2010;23 (07):770-776

24 Lee HY, Kim HU, Kim SS, et al. Surgical anatomy of the sphenopalatine artery in lateral nasal wall. Laryngoscope 2002;112(10): 1813-1818

25 Orhan I, Aydın S, Ormeci T, Yılmaz F. A radiological analysis of inferior turbinate in patients with deviated nasal septum by using computed tomography. Am J Rhinol Allergy 2014;28(01): e68-e72

26 Akoğlu E, Karazincir S, Balci A, Okuyucu S, Sumbas H, Dağli AS. Evaluation of the turbinate hypertrophy by computed tomography in patients with deviated nasal septum. Otolaryngol Head Neck Surg 2007;136(03):380-384

27 Ludlow JB, Ivanovic M. Comparative dosimetry of dental CBCT devices and 64-slice CT for oral and maxillofacial radiology. Oral Surg Oral Med Oral Pathol Oral Radiol Endod 2008;106(01): 106-114

28 Tyndall DA, Price JB, Tetradis S, Ganz SD, Hildebolt C, Scarfe WCAmerican Academy of Oral and Maxillofacial Radiology. Position statement of the American Academy of Oral and Maxillofacial Radiology on selection criteria for the use of radiology in dental implantology with emphasis on cone beam computed tomography. Oral Surg Oral Med Oral Pathol Oral Radiol 2012;113(06): 817-826

29 Gerbault O, Daniel RK, Kosins AM. The role of piezoelectric instrumentation in rhinoplasty surgery. Aesthet Surg J 2016;36 (01):21-34

30 Murchison AP, Pribitkin EA, Rosen MR, Bilyk JR. The ultrasonic bone aspirator in transnasal endoscopic dacryocystorhinostomy. Ophthal Plast Reconstr Surg 2013;29(01):25-29 